\title{
Adjuvant chemoradiation for resected gastric cancer: a 10-year experience
}

\author{
Amy T. Y. Chang $\cdot$ Wai Tong Ng $\cdot$ Ada L. Y. Law $\cdot$ \\ Ki Man Ku $\cdot$ Michael C. H. Lee $\cdot$ Anne W. M. Lee
}

Received: 12 June 2010/Accepted: 31 October 2010/Published online: 17 February 2011

(c) The International Gastric Cancer Association and The Japanese Gastric Cancer Association 2011

\begin{abstract}
Background The Intergroup 0116 study demonstrated that concurrent chemoradiation improved overall survival (OS) in resected gastric cancer. However, there are few reports focusing on late toxicity and factors governing prognosis. This study aimed to determine these two important aspects for employing this regimen.

Methods Patients with resected gastric cancer stage IB to IV (M0) disease, treated between July 1998 and December 2007, were analyzed. The majority of the patients were treated using 5 cycles of 5 -fluorouracil (5FU)/leucovorin chemotherapy with $45 \mathrm{~Gy} / 25$ fractions radiotherapy concurrent with cycles 2 and 3, as per the Intergroup 0116 study.

Results We treated 120 patients (107 standard protocol, 13 with concurrent $5 \mathrm{FU}$ alone), and $14 \%$ had a close or positive margin. Median age was 59 years (35-79 years). Acute toxicity $\geq$ grade 3 was seen in $66 \%$ of all patients (hematological $61 \%$, stomatitis $3 \%$, diarrhea $6 \%$, vomiting $2 \%$ ). Median follow-up was 33 months (range 6-125 months). Five-year OS and relapse-free survival were 51 and 54\%, respectively. On multivariate analysis, surgical margin status, stage of the disease, and radiotherapy with computed tomography (CT) planning were important prognostic
\end{abstract}

A. T. Y. Chang $(\bowtie) \cdot$ W. T. Ng • A. L. Y. Law •

K. M. Ku - A. W. M. Lee

Department of Clinical Oncology,

Pamela Youde Nethersole Eastern Hospital,

3 Lok Man Road, Chai Wan, Hong Kong

e-mail: tienyee.chang@gmail.com

M. C. H. Lee

Department of Medical Physics,

Pamela Youde Nethersole Eastern Hospital,

Chai Wan, Hong Kong factors. Anemia and gastritis were the two most frequently occurring late complications, though they were usually mild and asymptomatic. Clinically significant renal impairment was uncommon. Other rare complications included intestinal obstruction, malabsorption, hypertension, and secondary malignancy.

Conclusions Postoperative chemoradiation is safe and late toxicity is usually mild in extent. Results were comparable to the Intergroup 0116 study. R0 resection is of utmost importance and radiotherapy should best be delivered by conformal techniques.

Keywords Gastric neoplasm · Gastrectomy · Combined modality therapy $\cdot$ Toxicity $\cdot$ Prognosis

\section{Introduction}

Gastric carcinoma is the sixth most common cancer in Hong Kong [1] and surgery is the primary treatment. However, the overall 5-year survival rate following surgery alone remains low, at less than $40 \%$ [2]. Various adjuvant therapies are being explored in the treatment of gastric cancer. The Medical Research Council (MRC) Adjuvant Gastric Infusional Chemotherapy (MAGIC) trial, using perioperative chemotherapy (epirubicin, cisplatin, and 5-fluorouracil [5FU]), and the Japanese Adjuvant Chemotherapy Trial of S-1 for Gastric Cancer (ACTS-GC) study, have both demonstrated superior outcomes with the addition of adjuvant chemotherapy [3, 4]. Meta-analyses [5, 6], however, have revealed only a marginal impact on using adjuvant chemotherapy alone. Gunderson and Sosin [7] showed that relapse in gastric cancer patients after initial curative surgery consisted of local recurrence or regional lymph node metastasis in $87.8 \%$ of the patients. The high 
rate of locoregional tumor recurrence in gastric cancer favors the concept of including radiotherapy in adjuvant treatment [8]. More recent meta-analyses have also shown a statistically significant improvement in survival for adjuvant radiotherapy in resectable gastric cancer $[9,10]$. The United States Intergroup 0116 study was the first large randomized trial demonstrating a significant improvement in disease-free survival and overall survival (OS) using chemoradiotherapy postoperatively compared to surgery alone [11]. The encouraging results of postoperative chemoradiation have defined a new standard of care in the United States. However, the Intergroup 0116 protocol has not been universally accepted because of the risks of toxicity. In the original study, the combined modality treatment group had high rates of significant toxicity, with grade 3 and grade 4 toxicity rates of 41 and $32 \%$ of cases, respectively. The major gastrointestinal toxicity rate was $33 \%$. There was also a $1 \%$ reported rate of treatmentrelated death. Also, there was a limited report of late complication.

Since the Intergroup study, guidelines on radiotherapy for resected gastric cancer have been published, which mainly consist of conventional two-dimensional planning [12]. In the era of using conformal and even intensity modulated radiotherapy (IMRT) with respiratory-gated procedures in gastric cancer, more advanced techniques may have an impact on treatment outcome. The main aim of the present study was to explore the late toxicity and prognostic factors, using the Intergroup regimen, in a group of Chinese patients following gastric cancer surgery in a Hong Kong regional hospital. In addition, OS, disease-free survival, and patterns of relapse are also reported.

\section{Methods, patients, and materials}

In this retrospective study, eligibility criteria included all Chinese patients with resected gastric cancer stage IB to IV (M0) disease treated with radical intent between July 1998 and December 2007, with Eastern Cooperative Oncology Group (ECOG) performance status of $0-2$ and adequate major organ function. In contrast to the Intergroup 0116 study, R0 and R1 resections were all included. Patients with metastatic disease detected preoperatively or at operation were excluded. Altogether 120 patients were included in this study. All except two patients (with Epstein-Barr virus-related lymphoepithelial carcinoma) had histologically confirmed adenocarcinoma. The majority of patients (107 out of 120 patients) were treated using 5 cycles of 5FU/leucovorin chemotherapy (first, fourth, and fifth cycle using $5 F U 425 \mathrm{mg} / \mathrm{m}^{2}$ and folinic acid $20 \mathrm{mg} / \mathrm{m}^{2}$ for 5 days; second cycle using $5 \mathrm{FU} 400 \mathrm{mg} / \mathrm{m}^{2}$ and folinic acid $20 \mathrm{mg} / \mathrm{m}^{2}$ for 4 days, and third cycle using $5 \mathrm{FU}$
$400 \mathrm{mg} / \mathrm{m}^{2}$ and folinic acid $20 \mathrm{mg} / \mathrm{m}^{2}$ for 3 days) and 45 Gy/25 fractions concurrent with cycles 2 and 3, as per the Intergroup 0116 study. However, prior to January 2000, patients were treated with 3 days concurrent $5 \mathrm{FU}$ $380 \mathrm{mg} / \mathrm{m}^{2}$ alone during cycles 2 and 3 . The remaining first, fourth, and fifth cycles were the same as those described above. In view of the manageable toxicity and the absence of unusual treatment complications, the chemotherapy dose was escalated as per the Intergroup scheme. Dose modification was also standardized since January 2000; a $20 \%$ dose reduction was applied if stomatitis or diarrhea was grade two or if the absolute neutrophil count (ANC) was between 0.5 and $<1.0$; and a $30 \%$ dose reduction was applied in patients with grade 3 stomatitis/diarrhea or an ANC of less than 0.5.

Sixty-two patients $(52 \%)$ received radiotherapy using anterior-posterior (AP-PA) opposing portals covering the tumor bed, anastomosis, and the regional lymphatics. The field borders were adopted from a consensus report by Smalley et al. [12]. Typically the field covered the upper border of T10 down to the lower border of L3. The right border is $2 \mathrm{~cm}$ to the right of the T11-L1 vertebral bodies, and the left border is about $1.5 \mathrm{~cm}$ from the remaining gastric remnant. A left inferior corner shield is used to cover the lower pole of the left kidney. Fluoroscopy was used to confirm the coverage of the diaphragm. With the popularity of computed tomography (CT) simulation and the availability of resources, CT planning was performed in 58 patients $(48 \%)$ during the later part of the study. CT images with $5 \mathrm{~mm}$ slide thickness were acquired from $\mathrm{T} 7$ to L5. The target volumes including the gastric remnant, anastomosis, porta hepatis, splenic hilum, duodenal stump, adjacent esophagus, and the regional lymph nodes were delineated. Organs at risk, including the spinal cord, kidneys, liver, and heart were also contoured. AP-PA opposing fields with the same coverage as conventional techniques were applied, with field size designed to cover the target volumes adequately. Dose volume histograms (DVHs) of the organs at risk were also evaluated to ensure that their doses were within tolerance (at least $70 \%$ of one functioning kidney exposed to $<20 \mathrm{~Gy} ;<60 \%$ of the volume of liver exposed to $>30 \mathrm{~Gy}$; maximum spinal cord dose $<45 \mathrm{~Gy} ;<30 \%$ of the volume of whole heart received a dose of $>40 \mathrm{~Gy}$ ). In cases where the tolerance doses for the organs at risk were exceeded, conformal radiotherapy planning was used (large anterior, upper AP/PA and lower anterior + lateral opposing fields), based on a recent series by Ringash et al. [13]. The radiation dose was 45 Gy in 25 fractions over 5 weeks. No prophylactic antiemetic regimen was given, but an $\mathrm{H} 2$ blocker was recommended for patients with subtotal gastrectomy.

After the adjuvant chemoradiation, patients were followed up at 2 and 8 weeks post-treatment for toxicity 
assessment. Then they were seen every 3 months until 2 years, and every 6 months up to 5 years, then annually.

Patient survival was calculated from the date of definitive surgery until death and was determined by the Kaplan and Meier method, with SPSS V.12.0 (SPSS, Chicago, IL, USA). Patients alive at the time of the study report were censored at that time. Univariate analyses were performed. Statistically significant differences were determined using the log-rank test. Cox regression was performed for any significant prognostic factors detected on univariate analyses. Information was also collected on the site(s) of first relapse. Acute and late toxicity were scored using the Common Terminology Criteria for Adverse Events (CTCAE v3.0).

\section{Results}

\section{Patient characteristics}

Patient characteristics are shown in Table 1. Of the 120 patients treated, 107 received the standard protocol as per the Intergroup study, 13 with concurrent $5 \mathrm{FU}$ alone during irradiation; 99 (82.5\%) patients had gastric and 21 (17.5\%) had cardia/gastroesophageal primaries. The curative surgery performed for the patient population was: total gastrectomyin 41 patients (34\%) and subtotal gastrectomyin 71 patients $(59 \%) ; 8$ patients $(7 \%)$ underwent an extended procedure. Most surgical records did not specify the exact nature of nodal dissection; therefore, the number of nodes examined by the pathologist was taken as a surrogate. The numbers of nodes removed were: $0-10$ nodes, 11 patients (9\%); 11-20 nodes, 39 patients (33\%); and $>21$ nodes, 69 patients (58\%). One patient had surgery performed in China and the number of nodes examined was not specified. Most patients had unfavorable prognostic features, with 89 patients (74\%) having poorly differentiated histology and $54(45 \%)$ with $>\mathrm{T} 3$ primary tumors; 111 patients (93\%) had regional nodal involvement and 17 (14\%) had close/involved margins (4 close margins defined as $<2 \mathrm{~mm}$ and 13 positive margins).

\section{Treatment}

The median time from surgery to the commencement of adjuvant therapy was 32 days (range 13-101 days); 108 (90\%) patients completed the therapy as planned. Of the 12 who did not complete the therapy, one stopped due to disease progression. Five patients stopped because of prolonged marrow suppression and another two had persistently elevated alanine transaminase levels, leading to termination of the chemotherapy. Four patients refused completion of the chemotherapy because of other toxicities in preceding cycles. Toxicities experienced led to
Table 1 Patient characteristics $(n=120)$

\begin{tabular}{ll}
\hline Male:female & $78: 42$ \\
Median age, years (range) & $59(35-79)$ \\
Median follow-up, months (range) & $33(6-125)$ \\
Median interval between surgery and & $32(13-101)$ \\
adjuvant therapy, days (range) & \\
T stage, no. of patients (\%) & \\
1 & $4(3)$ \\
2 & $62(52)$ \\
3 & $50(42)$ \\
4 & $4(3)$ \\
N stage, no. of patients (\%) & \\
0 & $9(7)$ \\
1 & $56(47)$ \\
2 & $35(29)$ \\
3 & $20(17)$ \\
Tumor grade, no. of patients (\%) & \\
Well-differentiated & $1(1)$ \\
Moderate & $25(21)$ \\
Poor & $89(74)$ \\
EBV-related lymphoepithelial carcinoma & $2(2)$ \\
Not stated & $3(2)$ \\
Type of surgery, no. of patients (\%) & \\
Total gastrectomy & $52(52)$ \\
Subtotal gastrectomy & $58(48)$ \\
Nuther & \\
Margin, no. of patients (\%) & \\
Clear margin (R0) & $71(34)$ \\
Close/positive margin (R1) & $71(59)$ \\
\hline
\end{tabular}

$C T$ computed tomography, $E B V$ Epstein-Barr virus

chemotherapy dose reductions in 88 patients, dose delay in 105 patients, and 81 patients requiring both. However, all patients completed the radiotherapy without interruption.

Acute toxicity

The overall documented rate of grade 3 or greater toxicity was $66 \%$ (Table 2). The grade 4 toxicity rate was $22 \%$, consisting entirely of neutropenia; 2 patients had neutropenic fever requiring hospital admission and intravenous broad-spectrum antibiotics. The overall rate of grade 3 gastrointestinal toxicity was $8 \%$ and the overall rate of grade 3 and 4 hematological toxicity was $61 \% ; 85 \%$ of the patients lost weight during treatment, with a mean weight loss of $4.2 \mathrm{~kg}$ (range $0.2-11.4 \mathrm{~kg}$ ). There were no toxicityrelated deaths. 
Table 2 Major acute toxic effects of chemoradiotherapy $(n=120)$

\begin{tabular}{lccc}
\hline & Grade 2 & Grade 3 & Grade 4 \\
\hline Hemoglobin & 59 & 6 & 0 \\
Platelets & 4 & 1 & 0 \\
Neutropenia & 26 & 45 & 26 \\
Stomatitis & 16 & 4 & 0 \\
Vomiting & 9 & 2 & 0 \\
Diarrhea & 34 & 7 & 0 \\
\hline
\end{tabular}

\section{Survival and relapse}

All patients were followed up for survival. The median follow-up for the whole group was 33 months (range 6-125 months). In the 66 patients who were alive at the time of the last evaluation, the median follow up was 57 months. There were 54 deaths; 47 of them were related to disease recurrence while six were due to incidental causes. One death was possibly related to a late complication (see below). Overall, the 5-year survival rate was $51 \%$ and the median OS was 64 months (Fig. 1a).

The 5-year relapse-free survival (RFS) rate was 54\% and the median RFS was 85 months (Fig. 1b); 53 (44\%) patients relapsed during the follow-up period. Two of these 53 patients relapsed locoregionally as the first site of disease recurrence; $27(51 \%)$ patients relapsed with either peritoneal or systemic disease; and the remaining 24 patients relapsed both locoregionally and systemically.

\section{Prognostic factors}

Detailed analysis of the 17 patients with close/involved resection margins at surgery showed that 11 had relapsed and ten of them succumbed afterwards. Five of these patients had documented locoregional relapse at the time of recurrence. Comparison of this group of patients with the group of patients with clear surgical margins showed statistically significant differences in locoregional control (LRC; $p=0.03$ ), distant failure-free survival (DFFS; $p<0.01$ ), RFS (Fig. 2a, $p<0.01$ ), and OS (Fig. 2b, $p<0.01)$. Similarly, $\mathrm{T}$ stage, $\mathrm{N}$ stage, and group stage were also predictive of LRC, DFFS, RFS, and OS on univariate analyses, while no correlation could be drawn with sex, age group (below 60 years vs. 60 or above), poorly differentiated histology, location of tumor (proximal vs. distal location of the stomach cancer), or numbers of sampled nodes (0-19 vs. 20 or above). Tumor size (less than 5 vs. $5 \mathrm{~cm}$ or above) was found to be predictive of RFS ( $p=0.04)$ only. It was also intriguing to see that CT planning, compared with conventional planning, was
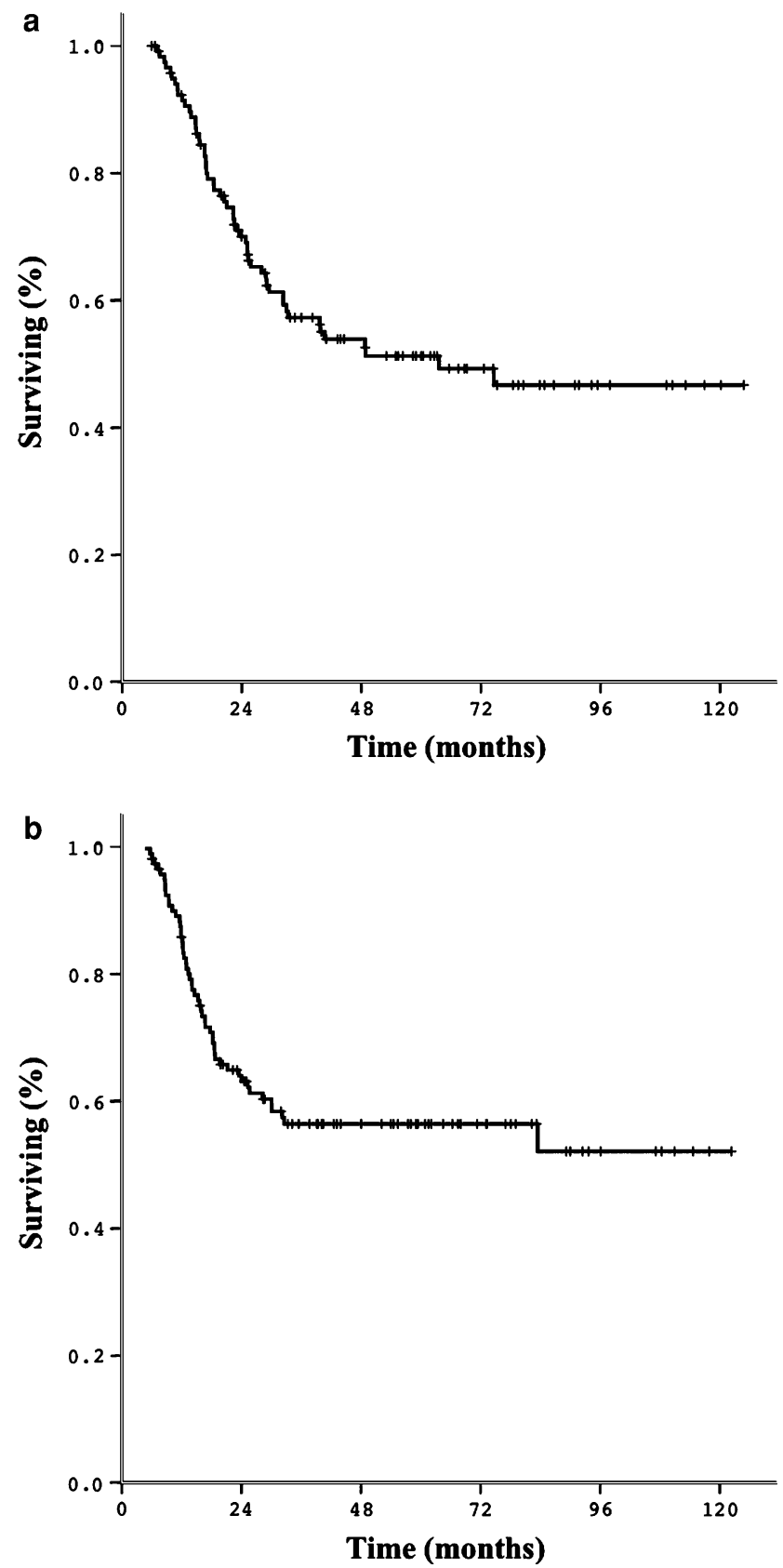

Fig. 1 Kaplan-Meier curves of overall survival (a) and relapse-free survival (b), $n=120$

associated with better RFS (Fig. 3a, $p=0.03$ ) and DFFS ( $p=0.05)$, but not with LRC $(p=0.26)$ or OS (Fig. 3b, $p=0.12$ ).

Multivariate analyses (Table 3) were carried out based on the following covariates: $\mathrm{T}$ stage, $\mathrm{N}$ stage, surgical margin status, and radiotherapy planning techniques (CT planning vs. conventional planning). Margin status remained the most important prognostic factor. However, radiotherapy planning techniques were also predictive of LRC, DFFS, RFS, and OS, as shown in Table 3. 

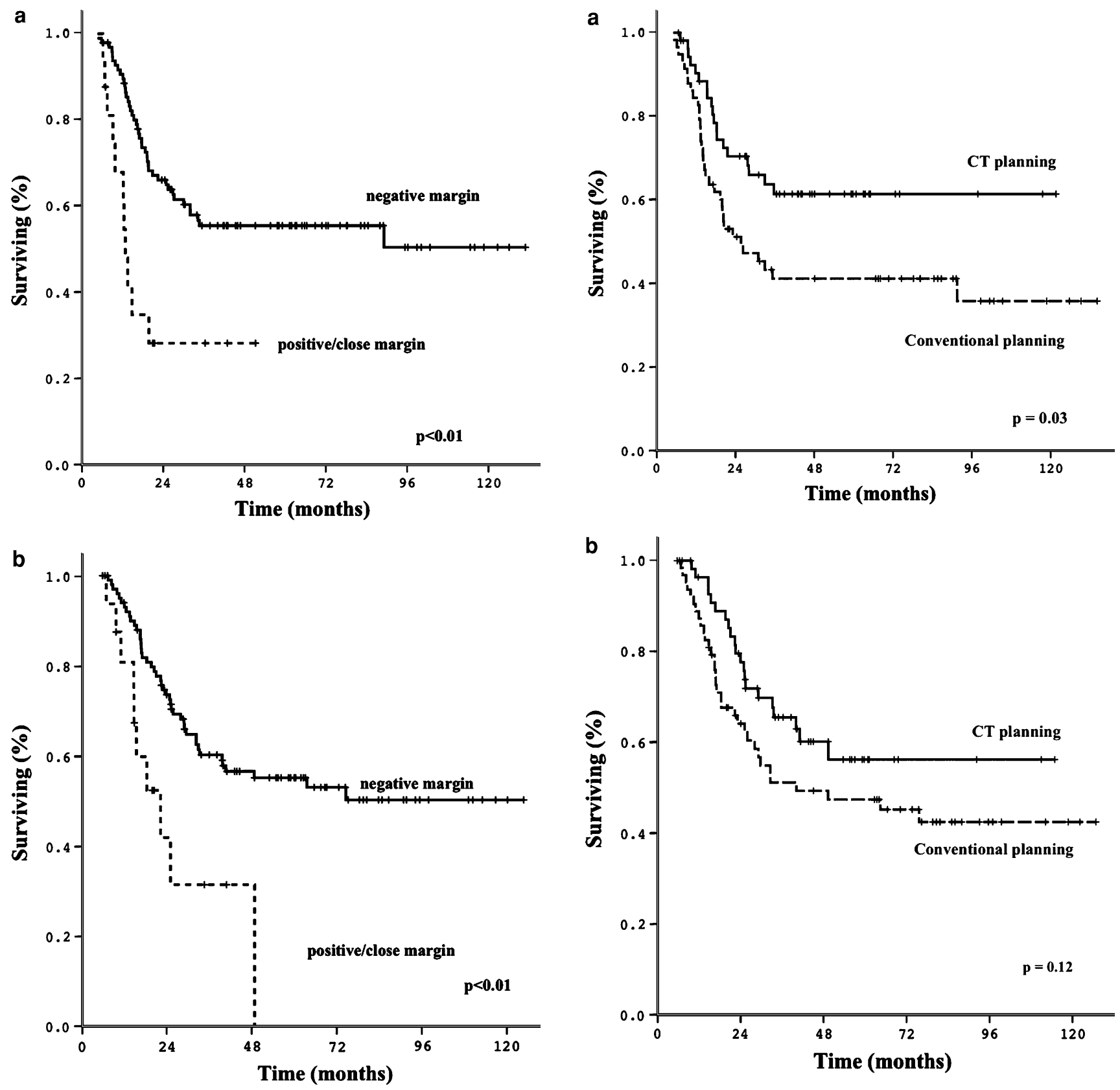

Fig. 2 Relapse-free survival curves (a) and overall survival curves (b) of close/positive resection margin versus clear margin

Fig. 3 Relapse-free survival curves (a) and overall survival curves (b) of conventional planning versus computed tomography (CT) planning

Late complications in surviving patients

Late complication data were available for 66 surviving patients after a median follow-up of 57 months (Table 4). Anemia was the most common finding and was found in 26 patients. During the time of follow-up, 8 patients suffered from grade 2 to 4 anemia (grade 2 in 4 patients, grade 3 in 3 patients, grade 4 in 1 patient), and two of them required blood transfusion. Seven of the 26 patients with anemia had associated gastritis, which was confirmed by esophagogastroscopy, while the other 19 patients had negative

endoscopic findings. At their most recent follow-up, the majority of these 26 patients had grade 1 anemia, except for one patient who had grade 2 anemia. Gastritis was the second most common finding, and was found in twelve patients. In 3 of these 12 patients, the gastritis was asymptomatic and was detected on surveillance esophagogastroscopy; the other patients had grade 2 gastritis, and mostly presented with epigastric pain, except for one patient who died of cerebrovascular accident precipitated by gastrointestinal bleeding. There were three cases of 
Table 3 Prognostic factors based on multivariate analyses $(n=120)$

\begin{tabular}{|c|c|c|}
\hline & Hazard ratio & $p$ value \\
\hline \multicolumn{3}{|l|}{ Overall survival } \\
\hline T stage & & 0.051 \\
\hline $\mathrm{T} 2$ vs. T1 & $9,765.65$ & 0.883 \\
\hline T3 vs. T1 & $10,547.53$ & 0.882 \\
\hline T4 vs. T1 & $47,755.27$ & 0.863 \\
\hline $\mathrm{N}$ stage & & 0.016 \\
\hline N1 vs. N0 & 2.77 & 0.184 \\
\hline N2 vs. N0 & 6.06 & 0.021 \\
\hline N3 vs. N0 & 5.51 & 0.027 \\
\hline Margin (close/positive margin vs. negative) & 3.94 & 0.001 \\
\hline Planning (CT vs. conventional planning) & 0.49 & 0.019 \\
\hline \multicolumn{3}{|l|}{ Relapse-free survival } \\
\hline $\mathrm{T}$ & & 0.014 \\
\hline $\mathrm{T} 2$ vs. $\mathrm{T} 1$ & 2.51 & 0.378 \\
\hline T3 vs. T1 & 3.00 & 0.289 \\
\hline T4 vs. T1 & 14.92 & 0.020 \\
\hline $\mathrm{N}$ & & 0.004 \\
\hline N1 vs. N0 & 7.63 & 0.053 \\
\hline N2 vs. N0 & 14.96 & 0.011 \\
\hline N3 vs. N0 & 19.06 & 0.005 \\
\hline Margin (close/positive margin vs. negative) & 4.68 & 0.000 \\
\hline Planning (CT vs. conventional planning) & 0.39 & 0.002 \\
\hline \multicolumn{3}{|l|}{ Locoregional control } \\
\hline $\mathrm{T}$ & & 0.012 \\
\hline T2 vs. T1 & 1.20 & 0.869 \\
\hline T3 vs. T1 & 0.76 & 0.802 \\
\hline T4 vs. T1 & 9.12 & 0.080 \\
\hline $\mathrm{N}$ & & 0.087 \\
\hline N1 vs. N0 & $20,721.47$ & 0.900 \\
\hline N2 vs. N0 & $70,550.97$ & 0.888 \\
\hline N3 vs N0 & $47,425.79$ & 0.892 \\
\hline Margin (close/positive margin vs. negative) & 6.88 & 0.001 \\
\hline Planning (CT vs. conventional planning) & 0.37 & 0.027 \\
\hline \multicolumn{3}{|l|}{ Distant failure-free survival } \\
\hline $\mathrm{T}$ & & 0.005 \\
\hline T2 vs. T1 & 2.35 & 0.414 \\
\hline T3 vs. T1 & 2.79 & 0.323 \\
\hline T4 vs. T1 & 16.46 & 0.016 \\
\hline $\mathrm{N}$ & & 0.010 \\
\hline N1 vs. N0 & 6.75 & 0.068 \\
\hline N2 vs. N0 & 13.74 & 0.014 \\
\hline N3 vs N0 & 15.00 & 0.010 \\
\hline Margin (close/positive margin vs. negative) & 5.76 & 0.000 \\
\hline Planning (CT vs. conventional planning) & 0.41 & 0.005 \\
\hline
\end{tabular}

anastomotic strictures, with one case requiring dilatation. Other gastrointestinal complications included two cases of malabsorption and one case of intestinal obstruction (the
Table 4 Summary of late toxicity $(n=66)$ based on Common Terminology Criteria for Adverse Events v3.0

\begin{tabular}{lclll}
\hline & Grade 1 & Grade 2 & Grade 3 & Grade 4 \\
\hline Anemia & 11 & 4 & 3 & 1 \\
Renal impairment & 3 & & & \\
Deranged liver function & 7 & & & \\
Gastritis & 3 & 9 & & \\
Intestinal obstruction & & & 1 & \\
Anastomotic stricture & & 3 & & \\
Malabsorption & 1 & 1 & 1 & \\
Hypertension & & & & \\
Secondary malignancy & & & &
\end{tabular}

One patient died of cerebrovascular accident precipitated by gastrointestinal bleeding

patient had a past history of bowel surgery for colon cancer).

Significant renal complication was uncommon. Two patients had mildly elevated serum creatinine (one patient had a background history of hypertension and diabetes mellitus), and one patient had membranous glomerulonephritis that manifested as proteinuria. Ultrasound or CT performed on these three patients showed atrophic left kidneys. Seven patients had mildly elevated liver enzymes, which in the majority of them, had returned to normal at their latest follow-up visit. One patient developed hypertension but it was uncertain whether this was related to previous radiation to the kidney and/or adrenal gland. There was also one case of secondary malignancy, in which the patient had diffuse large B-cell lymphoma of the stomach 5 years after treatment.

\section{Discussion}

Gastric cancer is one of the leading causes of cancer deaths, with more than 600,000 cases per year. While surgery with R0 resection is the only curative treatment, the prognosis remains poor, with a high risk of locoregional or distant relapse [14]. Therefore, a multimodal approach is essential for improving treatment outcome. Studies of adjuvant chemotherapy have not demonstrated consistent benefits, although several meta-analyses have shown only a small survival benefit [15-17]; most studies yielded negative findings when analyzed individually. The reasons for the conflicting results are that many trials were underpowered to detect clinical significance, and also suboptimal chemotherapy regimens (older schemes such as 5FU, adriamycin, and mitomycin-C combinations) were used $[14,18]$. Therefore, adjuvant chemotherapy alone is still not widely adopted worldwide. The United States Intergroup study (INT0116) has demonstrated a substantial 
benefit of adjuvant chemoradiation, and this has become the standard of care in the United States. In that study, 556 patients with resected gastric cancer were randomized between adjuvant chemoradiation with 5-fluorouracil $(5 \mathrm{FU}) /$ leucovorin versus observation. Results after 6 years of follow-up showed significant improvement in median OS (35 vs. 26 months, $p=0.006$ ) and disease-free survival (30 vs. 19 months, $p<0.001$ ) [19]. However, the study was criticized mainly due to suboptimal lymph node surgery, in which only $10 \%$ of patients received D2 dissections. Therefore, whether radiation is compensating for inadequate surgery, and whether adjuvant chemoradiation is equally beneficial for patients with extended lymphadenectomy (D2 or greater dissection) remains an unresolved question. Other drawbacks of the Intergroup study include few data on long-term complications, and the use of only conventional two-dimensional radiotherapy techniques, which may result in suboptimal coverage of target structures.

One of the primary aims of our study was to investigate the late toxicity when employing the Intergroup regimen. Our study revealed that anemia was the most common late toxicity, despite patients receiving regular vitamin B12 injections post-gastrectomy. Nevertheless, most cases were mild and insignificant, and were able to improve over time. Gastritis was also a frequent late complication, emphasizing the long-term need to prescribe adequate gastric protective agents such as $\mathrm{H} 2$ blockers. Renal toxicity is a serious complication of radiotherapy, as studies reveal gradual worsening of renal function of up to $50 \%$ at 18 months post-treatment. Factors contributing to renal impairment include the V20 of the left kidney (percentage volume of left kidney receiving more than $20 \mathrm{~Gy}$ ) and mean left kidney dose, and pre-radiotherapy creatinine clearance [20,21]. The results of the present study did not show significant clinically manifested renal complications; however, among the three patients with renal impairment, imaging did reveal atrophic left kidneys, which is clearly a radiation-induced effect. Other gastrointestinal complications including malabsorption, anastomotic stricture, and bowel obstruction also occurred occasionally. It would be helpful to further minimize the dose to the kidneys and small bowel with more sophisticated radiotherapy techniques.

Our study showed that the surgical margin and radiotherapy planning techniques, as well as the stage of disease, were important prognostic factors. In contrast to the Intergroup study, our study also included patients with R1 resection. The results concur with other international series in that $\mathrm{R} 0$ resection has a superior outcome compared with $\mathrm{R} 1$ or $\mathrm{R} 2$ resection [18]. It is not surprising that CT planning was a favorable predictor of survival, as, compared with conventional planning, it enabled optimal coverage of target structures, including the porta hepatitis and other regional lymph nodes, the duodenal stump, and the gastric remnant. In fact, $35 \%$ of the radiotherapy plans in the INT0116 study deviated from the protocol, reflecting the inadequacies of conventional techniques in target delineation.

However, due to the caveats of a retrospective study, one has to be reminded that the late complication rates were calculated based only on surviving patients, while recurrent cases were excluded, as it was difficult to differentiate between symptoms due to complications and those due to recurrence. The three cases of impaired renal function as a late complication were based on only serum creatinine, and a more accurate measurement using, e.g., a radioisotope assay would have been more preferable. There is also a risk of underestimating the incidence of local recurrence because esophagogastroscopy was not routinely performed in patients in whom distant recurrence was detected.

As mentioned earlier in this section, the role of chemoradiation after D2 dissection remains controversial. Several Asian studies have attempted to elucidate this question [22, 23]. A Korean group demonstrated the advantage of adjuvant chemoradiation in D2-resected gastric cancer [22]. In that observational study, 544 patients received postoperative chemoradiation (45 Gy concurrent with 5FU/leucovorin as per the INT0116 protocol) after curative D2 resection, and the results were compared with those of 446 patients who received surgery alone. The results showed a significantly prolonged median OS (95.6 vs. 62.6 months; hazard ratio [HR] 0.80, $p=0.02)$ and RFS (75.6 vs. 52.7 months; HR 0.80, $p=0.016)$ in the chemoradiation group. The cohort study by Leong et al. [23] of 70 patients who received D2 dissection and adjuvant chemoradiation demonstrated 3-year OS, disease-free survival, and local control (60.6, 54.1, and $84.3 \%$, respectively), comparable to the results of the Korean group [22]. The outcome from our present series is comparable to those of these other Asian studies, and is able to demonstrate superior survival results and lower acute gastrointestinal complication rates compared with the Intergroup 0116 study.

Despite D2 dissections being widely performed in Asian countries, the extent of lymphadenectomy remains controversial. Dutch and United Kingdom MRC study groups have conducted randomized trials to compare D1 versus D2 dissections; D2 dissection did not show any survival benefit, but there was increased postoperative mortality and morbidity [24]. On the contrary, a Japanese group was able to achieve superior survival results, low mortality, and low recurrence rates after extended lymph node dissections, and an Italian study also supported the benefits of D2 dissection, without added complications $[25,26]$. In the present 
study, due to the heterogeneity of the operative records, the exact D number was not specified. Thus, we were not able to confirm the role of applying the Intergroup regimen in the context of D2 dissection. This question is now being addressed by an ongoing phase III trial in Korea (ARTIST trial), which compares adjuvant chemotherapy alone versus chemoradiation in curative D2-resected gastric cancer [27].

Future studies may also aim at improved radiotherapy techniques. IMRT and image-guided radiotherapy (IGRT) have been demonstrated to be feasible approaches for delivering chemoradiation with improved dose coverage to the target volumes, and further sparing of organs at risk [28-31]. In our center, IGRT with respiratory control (either by gating or breath-hold) has been implemented routinely since 2008, aiming to reduce the effect of organ motion. Attempts to intensify the chemotherapy regimens have also been tested in several ongoing studies, including the CRITICS and CALGB 80101 trials, which combine perioperative or postoperative epirubicin, cisplatin, 5FU (or capecitabine) with 5FU (or capecitabine)-based radiotherapy $[32,33]$.

\section{Conclusions}

Our results demonstrate that postoperative chemoradiation in our local Chinese population is feasible, with survival outcomes comparable to those in other Asian series. There was no acute toxicity-related death and late complications were generally mild and manageable by simple measures. Margin status and radiotherapy techniques were important prognostic factors, emphasizing the need for meticulous surgery to achieve clear surgical resection, and more sophisticated radiotherapy techniques to optimize the therapeutic ratio.

Conflict of interest None.

\section{References}

1. Hong Kong Cancer Registry. Hong Kong Cancer Stat 2007. Hong Kong: Hospital Authority; 2009.

2. Hundahl SA, Phillips JL, Menck HR. The National Cancer Data Base Report on poor survival of U.S. gastric carcinoma patients treated with gastrectomy: Fifth Edition American Joint Committee on Cancer staging, proximal disease, and the "different disease" hypothesis. Cancer. 2000;88:921-32.

3. Cunningham D, Allum WH, Stenning SP, Thompson JN, Van de Velde CJ, Nicholson M, et al. Perioperative chemotherapy versus surgery alone for resectable gastroesophageal cancer. N Engl J Med. 2006;355:11-20.

4. Sakuramoto S, Sasako M, Yamaguchi T, Kinoshita T, Fujii M, Nashimoto A, et al. Adjuvant chemotherapy for gastric cancer with $\mathrm{S}-1$, an oral fluoropyrimidine. $\mathrm{N}$ Engl $\mathrm{J}$ Med. 2007;357:1810-20.
5. Gianni L, Panzini I, Tassinari D, Mianulli AM, Desiderio F, Ravaioli A, et al. Meta-analyses of randomized trials of adjuvant chemotherapy in gastric cancer. Ann Oncol. 2001;12:1178-80.

6. Panzini I, Gianni L, Fattori PP, Tassinari D, Imola M, Fabbri P, et al. Adjuvant chemotherapy in gastric cancer: a meta-analysis of randomized trials and a comparison with previous meta-analyses. Tumori. 2002;88:21-7.

7. Gunderson LL, Sosin H. Adenocarcinoma of the stomach: areas of failure in a re-operation series (second or symptomatic look) clinicopathologic correlation and implications for adjuvant therapy. Int J Radiat Oncol Biol Phys. 1982;8:1-11.

8. Klautke G, Fietkau R. Significance of radiation therapy for adenocarcinomas of the esophagus, gastroesophageal junction and gastric cancer with special reference to the MAGIC trial. Strahlenther Onkol. 2007;183:163-9.

9. Fiorica F, Cartei F, Enea M, Licata A, Cabibbo G, Carau B, et al. The impact of radiotherapy on survival in resectable gastric carcinoma: a meta-analysis of literature data. Cancer Treat Rev. 2007;33:729-40.

10. Valentini V, Cellini F, Minsky BD, Mattiucci GC, Balducci M, D'Agostino G, et al. Survival after radiotherapy in gastric cancer: systematic review and meta-analysis. Radiother Oncol. 2009;92:176-83.

11. Macdonald JS, Smalley SR, Benedetti J, Hundahl SA, Estes NC, Stemmermann GN, et al. Chemoradiotherapy after surgery compared with surgery alone for adenocarcinoma of the stomach or gastroesophageal junction. N Engl J Med. 2001;345:725-30.

12. Smalley SR, Gunderson L, Tepper J, Martenson JA Jr, Minsky B, Willett C, et al. Gastric surgical adjuvant radiotherapy consensus report: rationale and treatment implementation. Int $\mathrm{J}$ Radiat Oncol Biol Phys. 2002;52:283-93.

13. Ringash J, Khaksart SJ, Oza A, Couture J, Japp B, Moore M, et al. Post-operative radiochemotherapy for gastric cancer: adoption and adaptation. Clin Oncol ( $\mathrm{R}$ Coll Radiol). 2005; 17:91-5.

14. De Vita F, Vecchione L, Galizia G, Di Martino N, Fabozzi T, Catalano G, et al. Perspectives in adjuvant therapy of gastric cancer. Oncology. 2009;77(Suppl 1):38-42.

15. Earle CC, Maroun JA. Adjuvant chemotherapy after curative resection for gastric cancer in non-Asian patients: revisiting a meta-analysis of randomised trials. Eur $\mathrm{J}$ Cancer. 1999;35:1059-64.

16. Mari E, Floriani I, Tinazzi A, Buda A, Belfiglio M, Valentini M, et al. Efficacy of adjuvant chemotherapy after curative resection for gastric cancer: a meta-analysis of published randomised trials. A study of the GISCAD (Gruppo Italiano per lo Studio dei Carcinomi dell'Apparato Digerente). Ann Oncol. 2000;11: 837-43.

17. Janunger KG, Hafstrom L, Glimelius B. Chemotherapy in gastric cancer: a review and updated meta-analysis. Eur J Surg. 2002;168:597-608.

18. Lim L, Michael M, Mann GB, Leong T. Adjuvant therapy in gastric cancer. J Clin Oncol. 2005;23:6220-32.

19. Macdonald JS. Role of post-operative chemoradiation in resected gastric cancer. J Surg Oncol. 2005;90:166-70.

20. May KS, Khushalani NI, Chandrasekhar R, Wilding GE, Iyer RV, Ma WW, et al. Analysis of clinical and dosimetric factors associated with change in renal function in patients with gastrointestinal malignancies after chemoradiation to the abdomen. Int $\mathbf{J}$ Radiat Oncol Biol Phys. 2010;76:1193-8.

21. Jansen EP, Saunders MP, Boot H, Oppedijk V, Dubbelman R, Porritt B, et al. Prospective study on late renal toxicity following postoperative chemoradiotherapy in gastric cancer. Int $\mathrm{J}$ Radiat Oncol Biol Phys. 2007;67:781-5.

22. Kim S, Lim DH, Lee J, Kang WK, MacDonald JS, Park CH, et al. An observational study suggesting clinical benefit for adjuvant 
postoperative chemoradiation in a population of over 500 cases after gastric resection with D2 nodal dissection for adenocarcinoma of the stomach. Int J Radiat Oncol Biol Phys. 2005;63:1279-85.

23. Leong CN, Chung HT, Lee KM, Shakespeare TP, Mukherjee RK, Wong LC, et al. Outcomes of adjuvant chemoradiotherapy after a radical gastrectomy and a D2 node dissection for gastric adenocarcinoma. Cancer J. 2008;14:269-75.

24. Moehler M, Lyros O, Gockel I, Galle PR, Lang H. Multidisciplinary management of gastric and gastroesophageal cancers. World J Gastroenterol. 2008;14:3773-80.

25. Maruyama K, Sasako M, Kinoshita T, Sano T, Katai H, Hada M, et al. Should systematic lymph node dissection be recommended for gastric cancer? Eur J Cancer. 1998;34:1480-9.

26. Degiuli M, Sasako M, Calgaro M, Garino M, Rebecchi F, Mineccia M, et al. Morbidity and mortality after D1 and D2 gastrectomy for cancer: interim analysis of the Italian Gastric Cancer Study Group (IGCSG) randomised surgical trial. Eur J Surg Oncol. 2004;30:303-8.

27. Lee J, Kang W, Lim D, et al. Phase III trial of adjuvant capecitabine/cisplatin (XP) versus capecitabine/cisplatin/RT (XPRT) in resected gastric cancer with D2 nodal dissection (ARTIST trial): safety analysis. J Clin Oncol 2009;27(15 Suppl):abstr 4537.
28. Ringash J, Perkins G, Brierley J, Lockwood G, Islam M, Catton $\mathrm{P}$, et al. IMRT for adjuvant radiation in gastric cancer: a preferred plan? Int J Radiat Oncol Biol Phys. 2005;63:732-8.

29. Wieland P, Dobler B, Mai S, Hermann B, Tiefenbacher U, Steil $\mathrm{V}$, et al. IMRT for postoperative treatment of gastric cancer: covering large target volumes in the upper abdomen: a comparison of a step-and-shoot and an arc therapy approach. Int J Radiat Oncol Biol Phys. 2004;59:1236-44.

30. Milano MT, Garofalo MC, Chmura SJ, Farrey K, Rash C, Heimann $\mathrm{R}$, et al. Intensity-modulated radiation therapy in the treatment of gastric cancer: early clinical outcome and dosimetric comparison with conventional techniques. Br J Radiol. 2006;79:497-503.

31. Wysocka B, Kassam Z, Lockwood G, Brierley J, Dawson LA, Buckley CA, et al. Interfraction and respiratory organ motion during conformal radiotherapy in gastric cancer. Int $\mathbf{J}$ Radiat Oncol Biol Phys. 2010;77:53-9.

32. Randomized Phase III Trial of Adjuvant Chemotherapy or Chemoradiotherapy in Resectable Gastric Cancer (CRITICS). http://www.clinicaltrial.gov/ct2/show/NCT00407186?term=CRITI CS\&rank=3.

33. Chemotherapy and Radiation Therapy After Surgery in Treating Patients With Stomach or Esophageal Cancer (CALGB80101). http://www.clinicaltrial.gov/ct2/show/NCT00052910?term=CALG B80101\&rank=1. 